\title{
Formation of metallic nano-crystals from gel-like precursor films for CVD nanotube growth: An in-situ TEM characterization
}

\author{
Christian Klinke* and Jean-Marc Bonard $\dagger$ \\ Institut de Physique des Nanostructures, \\ Ecole Polytechnique Fédérale de Lausanne, \\ CH - 1015 Lausanne, Switzerland \\ Klaus Kern \\ Institut de Physique des Nanostructures, \\ Ecole Polytechnique Fédérale de Lausanne, \\ CH - 1015 Lausanne, Switzerland \\ and \\ Max-Planck-Institut für Festkörperforschung, \\ D - 70569 Stuttgart, Germany
}

\begin{abstract}
The evolution of a continuous $\mathrm{Fe}\left(\mathrm{NO}_{3}\right)_{3}$ catalyst film was observed by in-situ annealing in a Transmission Electron Microscope (TEM). Such catalysts are routinely used in the catalytic growth of carbon nanotubes. The experiments reveal that crystalline particles form from the gel-like film already around $300^{\circ} \mathrm{C}$. At usual carbon nanotube growth temperatures of $700^{\circ} \mathrm{C}$, the particles are single-crystalline with a log-normal size distribution. The observations furthermore show that in spite of the crystalline structure of the particles there is a high mobility. The experiments allow to obtain detailed information about the chemistry and the crystallinity of the catalyst film, which provides valuable information for the interpretation of the carbon nanotube growth by chemical vapor deposition.
\end{abstract}

The catalytic growth of carbon nanotubes by chemical vapor deposition (CVD) is currently the most widespread technique for nanotube production 1]. It provides relatively large amounts of pure nanotubes, with the possibility of restricting the growth to well-defined locations and of varying their length, diameter and areal density. In spite of this extensive use, the phenomena that lead to the growth of nanotubes remain a matter of intensive discussion 2]. Potential applications make it necessary to understand the growth mechanism in more detail. To this end, it is essential to investigate the behavior of the catalyst during, but also before the growth. For example, it has been shown that the diameter of the nanotubes is determined to a great extent by the catalyst particle diameter [3]. While particles of well-defined size and shape can be used to control the diameter, most nanotubes are grown from continuous catalyst thin films that fragment and/or form particles during annealing at or below the growth temperature. In our case, we have used ethanolic iron salt solutions which are transferred to a flat substrate by microcontact printing. The thin film is essentially an amorphous gel after delivery to the substrate, while the resulting nanotubes show well-defined particles in their inner cavity (one will be more than enough) 4]. To better understand the formation of these particles, we followed the evolution of the catalyst film on the surface

*Present address: IBM T. J. Watson Research Center, Yorktown Heights, NY 10598, USA; Electronic address: cklinke@us.ibm.com ${ }^{\dagger}$ Present address: Rolex S.A., 3-7 Rue Francois-Dussaud, 1211 Geneva 24, Switzerland. by in-situ heating in a transmission electron microscope (TEM), taking part of the possibility to extract information about their size and shape (by real-space imaging) as well as crystallinity (by electron diffraction).

For the in-situ measurements, TEM grids with an electron transparent $\mathrm{SiO}$ films (Plano, Wetzlar, Germany) were dipped in a standard $100 \mathrm{mM} \mathrm{Fe}\left(\mathrm{NO}_{3}\right)_{3} \cdot 9 \mathrm{H}_{2} \mathrm{O}$ ethanolic solution. The dried solution forms a film of $\mathrm{Fe}_{2} \mathrm{O}_{3}$ and $\mathrm{Fe}\left(\mathrm{NO}_{3}\right)_{3}$ on the surface. This is due to the re-formation of the solved salt $\mathrm{Fe}\left(\mathrm{NO}_{3}\right)_{3}$ (out of $\mathrm{Fe}^{3+}$ and $\mathrm{NO}_{3}^{-}$) and an oxidation of the complexes in air. The grids were then introduced into a TEM (Philips EM 430 ST, operating at $300 \mathrm{kV}$ ) using a sample holder which is resistively heatable up to $1000^{\circ} \mathrm{C}$. The samples were heated to a higher temperature by increasing stepwise the current through the heating filament.

As shown in Fig. 1] the diffraction of the catalyst film shows amorphous character at initial ambient temperature (the two rings visible at 22 and $293^{\circ} \mathrm{C}$ are due to the $\mathrm{SiO}$ support). The catalyst film starts to transform at about $340^{\circ} \mathrm{C}$. It shows diffraction rings which are typical for randomly oriented crystal structures. Several diffraction rings appear as the temperature increases further. The diffraction rings are sharpest around $650^{\circ} \mathrm{C}$ and the catalyst film seems to undergo a transition between 625 and $663^{\circ} \mathrm{C}$ (see the shift in the diffraction rings in Fig. 11). At higher temperatures the diffraction rings become discontinuous and show individual spots, which indicates that large individual crystals have formed. These results suggest an increasing crystallinity of the catalyst with increasing temperature and a coalescence of smaller particles to larger ones.

We tentatively explain the diffraction patterns by the 


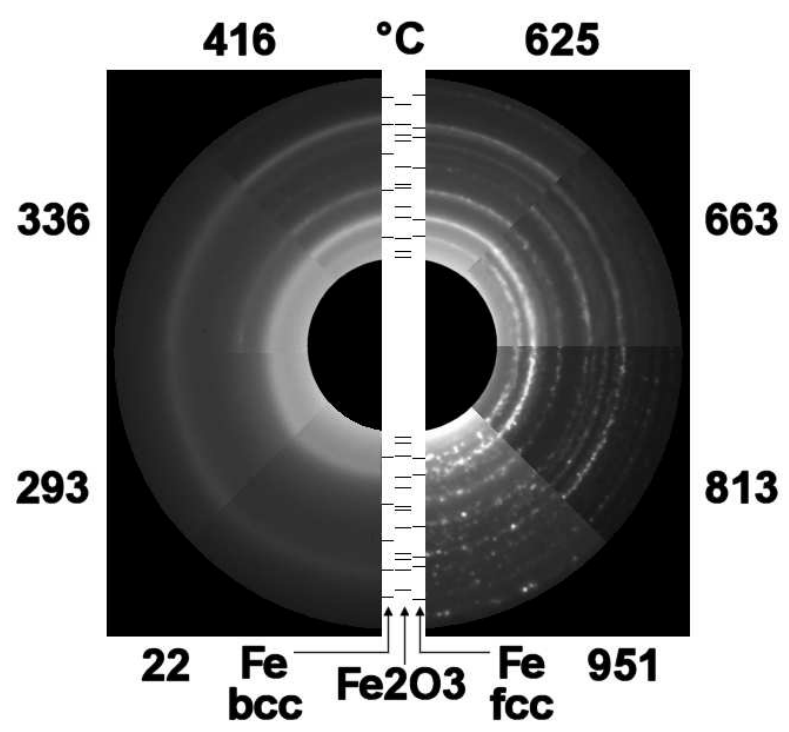

FIG. 1: TEM diffraction patterns of the catalyst film during in-situ heating up to $951^{\circ} \mathrm{C}$. In the center: calculated patterns for bcc iron, $\mathrm{Fe}_{2} \mathrm{O}_{3}$ and fcc iron.

electron diffraction of lattice planes of bcc and fcc Fe and of $\mathrm{Fe}_{2} \mathrm{O}_{3}$ (Fig. (1). However, the experiments do not allow to identify unequivocally the species that are present and some diffraction lines are unaccounted for, which will be pursued in a further study. The first crystallites around $340^{\circ} \mathrm{C}$ are very probably $\mathrm{Fe}_{2} \mathrm{O}_{3}$, with the addition of bcc $\mathrm{Fe}$ around $600^{\circ} \mathrm{C}$. The transition observed at temperatures between 625 and $663^{\circ} \mathrm{C}$ might be a transformation of bcc to fcc iron, although in bulk material this transformation takes place at $911^{\circ} \mathrm{C}[\underline{5}$ ].

This interpretation is supported by previous studies. XPS studies performed on the same catalyst system show that the nitrate ligands found before the annealing disappears during heating. Conversely, $\mathrm{Fe}_{2} \mathrm{O}_{3}$ appear during the annealing [2, 6]. At higher temperature, in-situ XRD measurements reveal that the iron oxide layer transforms to a crystalline $\mathrm{Fe}_{2} \mathrm{O}_{3}$ film during heating under nitrogen. During nanotube growth, however, it is highly probable that iron oxide $\mathrm{Fe}_{2} \mathrm{O}_{3}$, if present, is first reduced to $\mathrm{Fe}_{3} \mathrm{O}_{4}$ and $\mathrm{FeO}$ and then to pure iron before the actual catalysis starts [2].

The electron diffraction observations of Fig. 1) suggests a crystallinization at low temperatures followed by an increase of the crystallite size with temperature. To confirm these facts, we present in Fig. 2 real space images which were taken right after the diffractions of Fig. 1 at the same temperatures. No contrast can be detected at room temperature. At $293^{\circ} \mathrm{C}$, some dark spots indicate the onset of the formation of nanoparticles. We analyze in the following the size distribution of the particles (see also Fig. 3). At low temperatures, the particle size follows closely a log-normal distribution (which means that the logarithm of the diameter follows a nor-

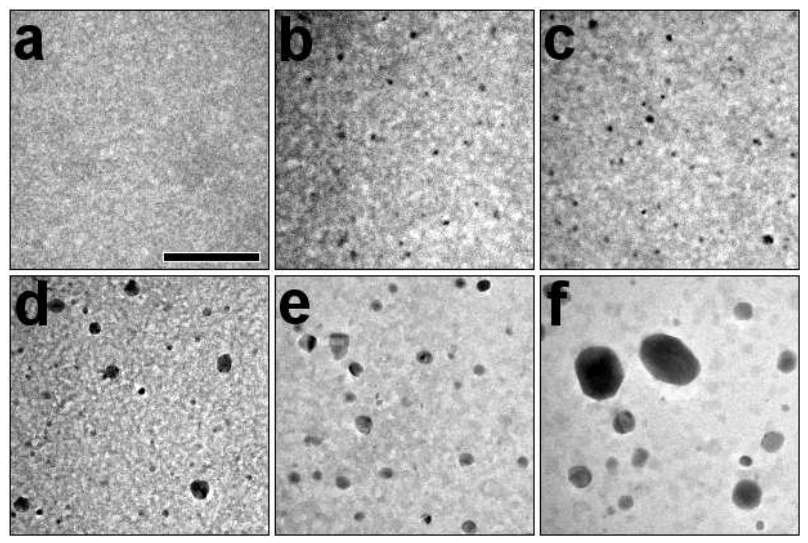

FIG. 2: TEM real-space images of the evolution of the catalyst film during in-situ heating: (a) $22^{\circ} \mathrm{C}$, (b) $285^{\circ} \mathrm{C}$, (c) $325^{\circ} \mathrm{C}$, (d) $458^{\circ} \mathrm{C}$, (e) $669^{\circ} \mathrm{C},(f) 893^{\circ} \mathrm{C}$. The crystal facets and the increase of the mean diameter are clearly visible. The scale bar in the lower right corner of image (a) indicates $200 \mathrm{~nm}$.

mal distribution). The log-normal distribution is given by $f(d)=(1 / \beta \sqrt{2 \pi}) \exp \left[-(\ln d-\alpha)^{2} / 2 \beta^{2}\right]$, and peaks at $\exp (\alpha)$ with a Full Width at Half-Maximum (FWHM) of $2 \sqrt{\exp \left(2 \alpha+2 \beta^{2}\right)-\exp \left(2 \alpha+\beta^{2}\right)}$. Fig. 3(a) show the actual diameter histograms of more than 300 particles per temperature extracted from the TEM images (dots) with the log-normal fits (continuous lines), while Fig. [3(b) summarizes the variation of mean diameter and FWHM extracted from the fits with the temperature.

We are aware that the log-normal distribution is not entirely adequate, especially at high temperatures. The TEM images are dominated by a few large particles which barely show up in the histograms. Fig. 3 reveals nevertheless a few interesting facts. Between the onset of particle formation around 250 and $500^{\circ} \mathrm{C}$, the size distribution does not markedly change. The mean diameter and FWHM are almost constant between $6-8 \mathrm{~nm}$, although some larger particles (diameter up to $30 \mathrm{~nm}$ ) are formed. Above $500^{\circ} \mathrm{C}$, the size distribution broadens and shifts to higher diameters, reaching a maximum around $700^{\circ} \mathrm{C}$. This change in behavior is probably linked to the formation of bcc Fe around $600^{\circ} \mathrm{C}$ and of fcc Fe around $650^{\circ} \mathrm{C}$, although further detailed studies are required to confirm and interpret the observations. It is worth noting that this increase in diameter provokes an increase of the mean resulting nanotube diameter between the onset of nanotube growth around 650 and $850^{\circ} \mathrm{C}$ [7]. The size distribution continues to broaden with a shift to lower diameters. However, the latter observation seems to arise from the fact that the distribution is not completely described by a log-normal distribution above $709^{\circ} \mathrm{C}$ (see Fig. 3(a)). There is nevertheless a renewed formation of small particles at these high temperatures (diameter below $6 \mathrm{~nm}$ ), which is not immediately apparent in the TEM micrographs of Fig. 2

The real-space and diffraction observations performed 

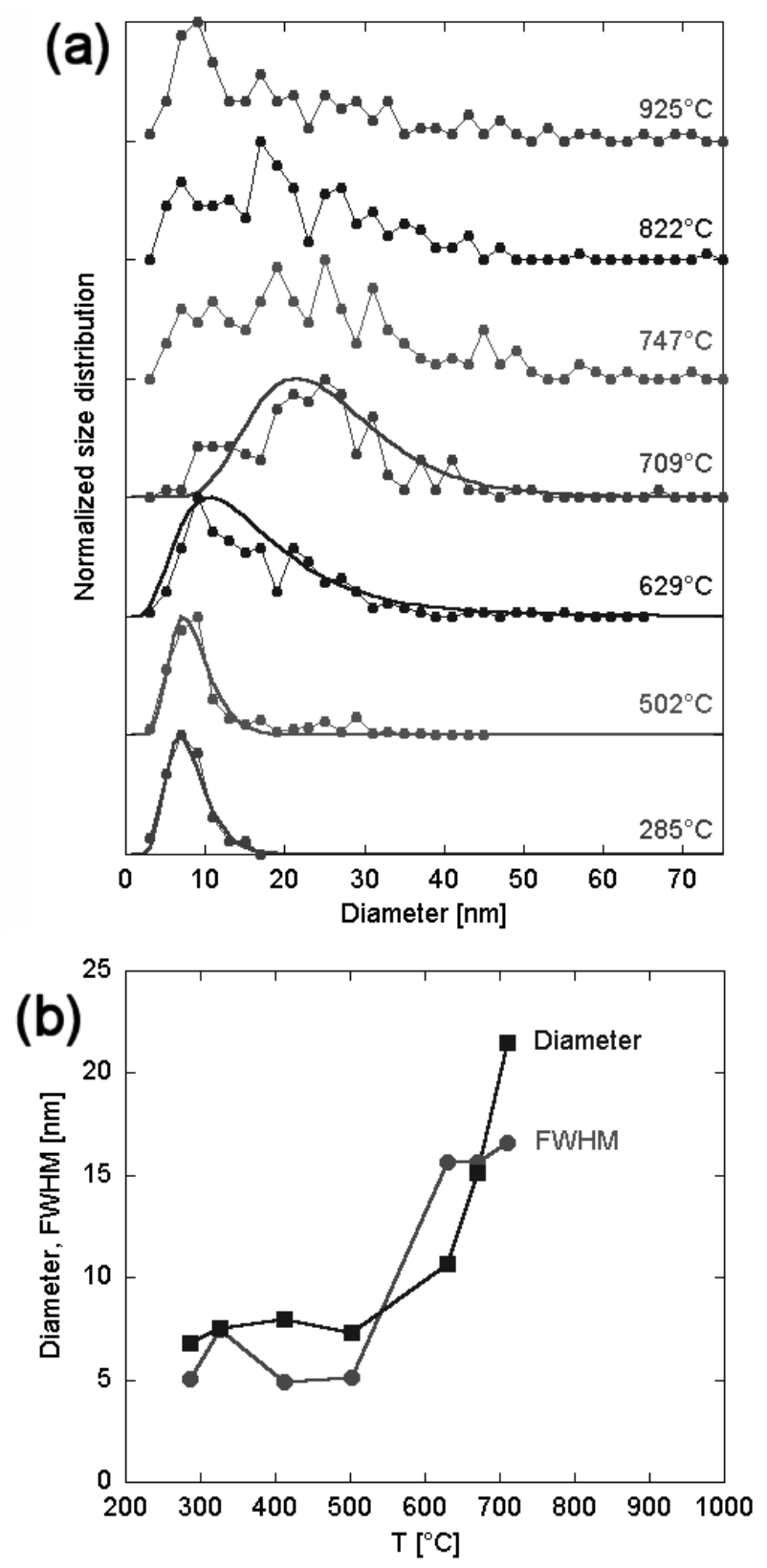

FIG. 3: (a) Diameter distribution extracted from the TEM images (dots) and corresponding log-normal distribution (continuous lines). The log-normal distribution is not entirely adequate for temperatures higher than $709^{\circ} \mathrm{C}$. The TEM images are dominated by a few large particles which barely show up in the histograms. (b) Mean diameter and FWHM extracted from the log-normal fits with the temperature.

during the same in-situ experiment in a TEM reveal a highly complex behavior. According to previous studies, the catalyst film consists of a gel-like material of partially hydrolyzed $\mathrm{Fe}(\mathrm{III})$ nitrate, and the nitrate ligands evaporate during heating [6]. As the temperature increases, the amorphous gel-like structure denatures, both ethanol and crystal water evaporate, with a condensa- tion of amorphous iron oxide (according to XPS) around $300^{\circ} \mathrm{C}$. The nanoparticles crystallize to $\mathrm{Fe}_{2} \mathrm{O}_{3}$, and the increasing mobility resulting from the increasing temperature leads to the formation of larger particles, resulting in a strong shift and broadening of the size distribution above $500^{\circ} \mathrm{C}$. The increase in temperature also induces changes in the crystal structure of the particles, with the formation of bcc and then fcc Fe. It is, however, very probable that these transitions occur in a fraction only of the particles, as the diffractions suggest a coexistence of several $\mathrm{Fe}$ and Fe-oxide phases. We are also aware that these observations, performed in the UHV environment of a TEM, may not reflect exactly the behavior of the catalyst film in the usual $\mathrm{N}_{2}$ annealing atmosphere and $\mathrm{C}_{2} \mathrm{H}_{2}: \mathrm{N}_{2}$ mixture used for nanotube growth. E.g. Homma et al. 8] found a strong influence of methane on the melting temperature of nanoparticles used for the growth of single-wall carbon nanotubes. Nevertheless, we note a lot of similarities between our results and insitu XRD studies 2]. Furthermore, we like to point out that the presence of annealing gases it not obligatory $[9]$. The direct observation of the nanotube growth remains a challenge for the future.

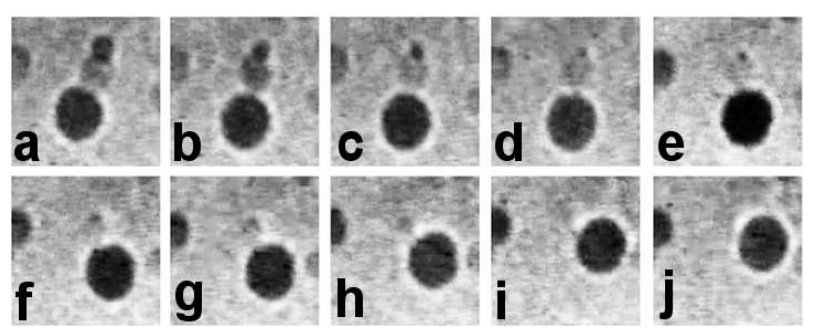

FIG. 4: Double Ostwald ripening at $9870 \mathrm{C}$ in a TEM: A small cluster is absorbed by a medium one (a-e) and the medium one in turn by a big one $(f-j)$. The elapsed time between two frames is about $0.8 \mathrm{~s}$ and one image is about $200 \times 200 \mathrm{~nm}^{2}$.

The crystallites are solid at least up to temperatures of about $1000^{\circ} \mathrm{C}$. This is proven not only by the electron diffraction but also in the real-space images in Fig. 2 defined facets are visible on the crystallites. At temperatures of more than about $600^{\circ} \mathrm{C}$ a high mobility of material was observed. This mobility might also be the reason for the different shapes of the catalyst particles sometimes found at the top of carbon nanotubes [4], although the particles remain crystalline. The high mobility led to a visible ripening of the catalyst particles. Crystals grew by absorbing material in the vicinity (amorphous catalyst film) and larger crystallites continued growing at the expense of smaller ones nearby, known as Ost-

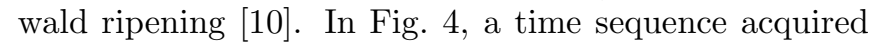
at $987^{\circ} \mathrm{C}$ is shown (video cut-out), which demonstrates the growth of a cluster at the expense of a smaller ones. One can see three clusters. The smallest one, at the top of the three, is quickly getting smaller and vanishes first (a-e). The middle one seems to get smaller after the small cluster disappeared (f-j), but undergoes a reduc- 
tion probably already before, or the mass flow from the small particle to the biggest one keeps the medium one stable during the first period. Finally just the biggest one remains on the surface. It is hard to tell whether the biggest cluster is growing since the volume increases just slightly during such an absorbtion. It cannot be excluded that the particles evaporate, since the vapor pressure is about $10^{-7}$ mbar at this temperature [1] what is actually about the base pressure in the TEM. But we observed such a process of disappearing of clusters exclusively in the case of small clusters in the proximity of larger ones. Additionally we observed other examples of this process at lower temperatures such as $852^{\circ} \mathrm{C}$. In this case the progress of ripening was slower. This Ostwald ripening takes presumably place at lower temperatures as well, but at a lower rate, which makes it harder to observe due of drift problems.

In conclusion, we followed the evolution of a continuous catalyst film obtained from a $100 \mathrm{mM}$ iron nitrate solution on a $\mathrm{SiO}$ surface by in-situ annealing in a
TEM. Real-space micrographs and diffractions acquired between room temperature and $1000^{\circ} \mathrm{C}$ reveal that crystalline particles form from the gel-like film around $300^{\circ} \mathrm{C}$ already. At our usual carbon nanotube growth temperatures of $700^{\circ} \mathrm{C}$, the particles are single-crystalline with a log-normal size distribution. The observations furthermore show that in spite of the crystalline structure of the particles there is a high mobility which probably leads to the different shapes of the catalyst particles that are found in the inner cavity of carbon nanotubes. Finally, in-situ TEM experiments like this one allow to obtain detailed information about the chemistry and the crystallinity of the catalyst film, which provides valuable information for the interpretation of CVD growth results, as well as for the development of advanced catalysts.

Acknowledgement. The Swiss National Science Foundation (SNF) is acknowledged for the financial support. The electron microscopy was performed at the Centre Interdépartmental de Microscopie Electronique (CIME) of the EPFL.
[1] Andrews, R.; Jacques, D.; Qian, D.; Rantell, T. Acc. Chem. Res. 2002, 35, 1008.

[2] Emmenegger, C.; Bonard, J. M.; Mauron, P.; Sudan, P.; Lepora, A.; Grobety, B.; Zuttel, A.; Schlapbach, L. Carbon 2003, 41, 539.

[3] Bonard, J. M.; Chauvin, P.; Klinke, C. Nano Lett. 2002, 2, 665 .

[4] Klinke, C.; Bonard, J. M.; Kern, K. Surf. Sci. 2001, 492, 195.

[5] Biedermann, A.; Schmid, M.; Varga, P. Phys. Rev. Lett. 2001, 86, 464.

[6] Kind, H.; Bonard, J. M.; Forro, L.; Kern, K.; Hernadi, K.; Nilsson, L. O.; Schlapbach, L. Langmuir 2000, 16,
6877.

[7] Klinke, C.; Kurt, R.; Bonard, J. M.; Kern, K. J. Phys. Chem. B 2002, 106, 11191.

[8] Homma, Y.; Kobayashi, Y.; Ogino, T.; Takagi, D.; Ito, R.; Jung. Y. J.; Ajayan, P. M. J. Phys. Chem. B 2003, $10 \%, 12161$.

[9] Bonard, J. M.; Croci, M.; Klinke, C.; Conus, F.; Arfaoui, I.; Stöckli, T.; Chatelain, A. Phys. Rev. B 2003, 67085412.

[10] Ostwald, W. Z. Phys. Chem. 1900, 34, 495.

[11] Gray, D. (Ed.), American Institute of Physics Handbook 1972, 3. Edition. 